\title{
Amended Description of the Genus Kineosporia, Based on Chemotaxonomic and Morphological Studies
}

\author{
TAKASHI ITOH, ${ }^{1+*}$ TAKUJI KUDO, ${ }^{1}$ FRANCESCO PARENTI,${ }^{2}$ AND AKIO SEINO ${ }^{1}$ \\ Japan Collection of Microorganisms, RIKEN, Wako-shi, Saitama 351-01, Japan, ${ }^{1}$ \\ and Lepetit Research Center, Gerenzano, Italy ${ }^{2}$
}

\begin{abstract}
The description of the genus Kineosporia is amended after chemotaxonomic and morphological studies of the type strain of the type species, Kineosporia aurantiaca JCM 3230. This organism yielded both LL- and meso-diaminopimelic acids, which suggested that the former is present in the mycelium and the latter is present in the spores. There was no characteristic sugar pattern. A diagnostic phospholipid was phosphatidylcholine, and a major menaquinone component was $\mathrm{MK}-9\left(\mathrm{H}_{4}\right)$. No iso/anteiso branched fatty acids and no mycolic acids were observed. Colonies on agar lacked aerial mycelia, formed central projections including spores, and were occasionally accompanied by bunches of spore clusters in the agar. Spores were catenated or were located singly or aggregately at the tips of the vegetative hyphae. Our data indicate that the strain representative of the genus Kineosporia shows some similarity to the spore dome actinomycetes described by Willoughby and by Makkar and Cross.
\end{abstract}

The name Kineosporia aurantiaca was proposed by Pagani and Parenti in 1978 (11) for an actinomycete that lacked aerial mycelia and bore numerous sporangia, each of which contained a single zoospore, on the substrate mycelia. Analyses of the cellular components in the original description showed the presence of glycine and LL-diaminopimelic acid $\left(\mathrm{A}_{2} \mathrm{pm}\right)$ in the cell walls and arabinose, galactose, and xylose in the whole organism. Pagani and Parenti emphasized the structure of the sporangium of their strain, compared with the sporangia of the other sporangium-forming actinomycetes, and consequently placed it in the new genus Kineosporia.

Recently, Hasegawa et al. (4) reported that whole cells of $K$. aurantiaca contain two isomers of $\mathrm{A}_{2} \mathrm{pm}$, the $\mathrm{LL}$ and meso forms, but lack arabinose and xylose. Our analyses agree with the analyses of these authors. In addition, we observed that the spores are catenated or form aggregately at the tips of hyphae.

Therefore, the description of the genus Kineosporia should be revised, and further studies on $K$. aurantiaca should be undertaken. We studied this organism to gain a better understanding of the taxonomy of the monospecific genus Kineosporia.

\section{MATERIALS AND METHODS}

Strain used, cultivation, and spore collection. $K$. aurantiaca JCM $3230^{\mathrm{T}}\left(=\mathrm{KCC} \mathrm{A-0320^{ \textrm {T } } = \mathrm { A } / 1 0 3 1 2 \text { Pagani and Parenti }}{ }^{\mathrm{T}}\right.$ ) ( $\mathrm{T}=$ type strain) was cultivated in yeast extract-glucose broth containing $10 \mathrm{~g}$ of yeast extract (Difco Laboratories, Detroit, Mich.) and $10 \mathrm{~g}$ of glucose in $1,000 \mathrm{ml}$ of distilled water ( $\mathrm{pH} \mathrm{7.2)}$ at $28^{\circ} \mathrm{C}$ for 5 to 6 days and then harvested by centrifugation. The biomass consisted of mycelial mats and numerous zoospores; this preparation was designated whole cultured organism.

Spores were collected from a spread culture grown on a

\footnotetext{
* Corresponding author.

$\dagger$ Present address: Central Research Laboratories, Kaken Pharmaceutical Co., Ltd., 2-28-8, Honkomagome, Bunkyo-ku, Tokyo 113, Japan.
}

dilute yeast extract-starch agar plate containing $0.5 \mathrm{~g}$ of yeast extract (Daigo Eiyo, Osaka, Japan), $2.5 \mathrm{~g}$ of soluble starch, and $15 \mathrm{~g}$ of agar in $1,000 \mathrm{ml}$ of distilled water ( $\mathrm{pH} \mathrm{7.3)}$ at $25^{\circ} \mathrm{C}$ for 6 days. The spread culture was dipped in distilled water, and the surface was lightly scratched to promote the release of motile spores. The resulting spore suspension was passed through filter paper (no. 2; Toyo Roshi, Tokyo, Japan), and the spores in the filtrate were collected by centrifugation at $10,000 \times g$ for $15 \mathrm{~min}$. The precipitate of spores was designated the spore fraction. Light microscopic observation indicated that the filtrate was free from mycelia.

Amino acid composition of cell walls and $A_{2}$ pm and sugar analyses. Cell wall peptidoglycan was prepared and hydrolyzed as described by Kawamoto et al. (6), and its amino acid composition was determined by using an automatic amino acid analyzer (model 835; Hitachi Ltd., Tokyo, Japan). The isomers of $\mathrm{A}_{2} \mathrm{pm}$ and the whole-organism sugar pattern were examined by using the methods of Staneck and Roberts (14).

Lipid analyses. Phospholipids were extracted from lyophilized cells with chloroform-methanol $(2: 1, \mathrm{vol} / \mathrm{vol})$, purified, and identified as described by Lechevalier et al. (7). In this procedure, menaquinones eluted with chloroform from the silica gel column were collected and partially purified by thin-layer chromatography, using a Merck Kieselgel $60 \mathrm{~F}_{254}$ plate developed with $n$-hexane-diethyl ether $(9: 1$, vol/vol). Menaquinone composition was determined by high-performance liquid chromatography as described by Tamaoka et al. (17). Methanol-isopropanol (2:1, vol/vol) was used as an eluent. Cellular fatty acid composition was determined by applying the methyl esters prepared by the methanolysis method of Ikemoto et al. (5) to a gas-liquid chromatograph (model GC-7A; Shimadzu Corp., Kyoto, Japan) equipped with a OV-1 capillary column $(0.24 \mathrm{~mm}$ by $50 \mathrm{~m})$ at $200^{\circ} \mathrm{C}$ and a flame ionized detector. The carrier gas was nitrogen. Gas-liquid chromatograms were analyzed by using a Shimadzu model C-R4A chromatograph-data processor. Mycolic acid was detected as described by Toriyama et al. (19).

Determination of deoxyribonucleic acid base composition. Deoxyribonucleic acid was extracted and purified by the phenol method of Saito and Miura (12). The base composi- 
TABLE 1. Cell wall and whole-cell sugar compositions of $K$. aurantiaca JCM $3230^{\mathrm{T}}$

\begin{tabular}{|c|c|c|c|c|c|c|c|c|c|c|c|c|c|}
\hline \multirow{2}{*}{ Prepn } & \multicolumn{4}{|c|}{$\begin{array}{l}\text { Amino acid composition of } \\
\text { peptidoglycan }^{a}\end{array}$} & \multicolumn{2}{|c|}{$\begin{array}{l}\text { Isomer of } \\
\mathrm{A}_{2} \mathrm{pm}\end{array}$} & \multicolumn{7}{|c|}{ Sugar composition of whole cells } \\
\hline & $\begin{array}{l}\text { Glutamic } \\
\text { acid }\end{array}$ & Alanine & $\mathrm{A}_{2} \mathrm{pm}$ & Glycine & LL & meso & Arabinose & Galactose & Glucose & Madurose & Mannose & Ribose & Xylose \\
\hline Whole cultured organism ${ }^{b}$ & 1.0 & 1.6 & 0.9 & 0.5 & + & + & - & + & + & - & + & + & - \\
\hline Spore fraction ${ }^{c}$ & 1.0 & 1.6 & 0.9 & 0.2 & - & + & - & $?^{d}$ & + & - & $\operatorname{Tr}$ & + & - \\
\hline
\end{tabular}

${ }^{a}$ Molar ratios, calculated by defining the amount of glutamic acid as 1.0 .

${ }^{b}$ Cells, including mycelial mats and numerous zoospores, were collected from a liquid culture.

$c$ Spores were collected from a spread culture cultivated on an agar plate.

${ }^{d}$ Galactose could not be detected due to the tailing of glucose on the thin-layer chromatogram.

tion was determined by the high-performance liquid chromatography method described by Tamaoka and Komagata (18).

Morphological characterization. We observed the morphological properties of the organism grown on oatmeal agar (20) and water agar at $28^{\circ} \mathrm{C}$. An agar block on which the culture grew was fixed with $1 \%$ glutaraldehyde and $1 \%$ osmium tetroxide; this was followed by washing with distilled water, dehydration through a graded ethanol series, transfer into amylacetate, and finally critical-point drying from liquid carbon dioxide. Alternatively, an agar block which was conductive stained as described by Watanabe et al. (21) was simply dried. The specimens were coated with gold by using an ion coater (model IB3; Eiko, Tsukuba, Japan) and were observed under a scanning electron microscope (model S-430; Hitachi Ltd.). The flagellated spores suspended in water were placed on collodion-coated grid, shadowed with platinum-palladium, and examined with a transmission electron microscope (model H-300; Hitachi Ltd.)

\section{RESULTS}

Chemotaxonomic characteristics. The cell wall compositions, isomers of $\mathrm{A}_{2} \mathrm{pm}$, and whole-cell sugar patterns of the whole cultured organism and the spore fraction are shown in Table 1 . The ratios of the contents of glutamic acid, alanine, and $\mathrm{A}_{2} \mathrm{pm}$ in the two peptidoglycans were similar to each other; however, the glycine content of the spore fraction was less than that of the whole cultured organism. The whole cultured organism contained LL- and meso- $\mathrm{A}_{2} \mathrm{pm}$ in comparable amounts and ribose, mannose, glucose, and galactose. In contrast, the spore fraction contained meso- $\mathrm{A}_{2} \mathrm{pm}$, ribose, and glucose, while LL- $\mathrm{A}_{2} \mathrm{pm}$ was not detected and mannose was present in small amounts. A large amount of glucose in the spore fraction prevented the detection of galactose on the thin-layer chromatogram.

Phosphatidylcholine was detected as a diagnostic phospholipid and was accompanied by diphosphatidylglycerol, phosphatidylinositol, and phosphatidylinositol mannoside (type PIII pattern of Lechevalier et al. [7]). The major component $(79 \%)$ of the isoprenoid quinone fraction was a tetrahydrogenated menaquinone with nine isoprene units [MK-9 $\left.\left(\mathrm{H}_{4}\right)\right]$, and small amounts of MK-8( $\left.\mathrm{H}_{4}\right)$ and MK-9 $\left(\mathrm{H}_{6}\right)$ were also present. The predominant cellular fatty acids were hexadecanoic acid $(16: 0 ; 27 \%)$, hexadecenoic acid $(16: 1$; $10 \%)$, octadecenoic acid $(18: 1 ; 23 \%)$, and 10 -methyl octadecanoic acid (10Me 19:0; 25\%); 2-hydroxyhexadecanoic acid (2OH 16:0) was also present (4\%); and iso/anteiso branched fatty acids and mycolic acids were absent. The guanineplus-cytosine content of the deoxyribonucleic acid was 69.2 mol\%. The phospholipid pattern, menaquinone composition, and cellular fatty acid profile of the spore fraction were similar to those of the whole cultured organism described above.
Morphological properties. The following morphological characteristics of the organism agreed with those of the original description: colonies on oatmeal agar were orange with a glossy appearance, lacked aerial mycelia, and formed central projections having a conical crateriform of cerebriform shape; numerous zoospores were released from the surfaces of colonies immersed in water; single spores were attached to the ends of vegetative hyphae at the edges of colonies (Fig. 1); and a sheath was present surrounding the spores. Under scanning electron microscopic observation, a gelatinous matrix was separated from the colony surface, and the central projections seemed to show hyphae radiating toward the surfaces (Fig. 2). In addition, we observed catenated spores around the central projections. Although most of the colonies on water agar formed the central projections with radiating vegetative hyphae, some colonies had small dome projections that bore a number of spore clusters with sporophores, which were bunched together in the agar (Fig. 3). A spore cluster observed on oatmeal agar is shown in Fig. 4. The zoospores released from the surfaces of colonies bore polar tufts of flagella (Fig. 5).

\section{DISCUSSION}

$K$. aurantiaca was first described as an actinomycete having cell wall type I containing glycine and LL- $\mathrm{A}_{2} \mathrm{pm}(8)$, and its whole cell sugar pattern was peculiar because of the presence of arabinose, galactose, and xylose. However, in this study we demonstrated that this organism yields two isomers of $\mathrm{A}_{2} \mathrm{l}$ without a characteristic sugar pattern (8), as reported by lasegawa et al. (4).

The whole cultured organism contained the two $A_{2} p m$ isomers, and the spore fraction contained only meso- $\mathrm{A}_{2} \mathrm{pm}$. Furthermore, the glycine content of the purified peptidoglycan of the spore fraction was less than that of the whole cultured organism. These data suggest that the mycelium peptidoglycan contains glycine and $L L-A_{2} p m$, while the spore peptidoglycan contains meso- $\mathrm{A}_{2} \mathrm{pm}$. We presume that the previous data on this cell wall component depended on the analysis of a culture yielding few spores, possibly due to the medium composition and the incubation time (Emerson medium and $72 \mathrm{~h}$ of incubation). Although the sugar composition is changeable, at least in the mannose content, the detection of arabinose and xylose described in the original paper is not accounted for by the distribution of the spores and mycelium in the preparation. We could not find any good reasons for the discrepancy.

A spore enclosed by a thin outer sheath was called a sporangium by Pagani and Parenti (11), who compared this structure with the sporangia of the other sporangiumforming actinomycetes. Although such a morphological comparison is effective for identification of the organism, the term sporangium as applied to actinomycetes is not well 


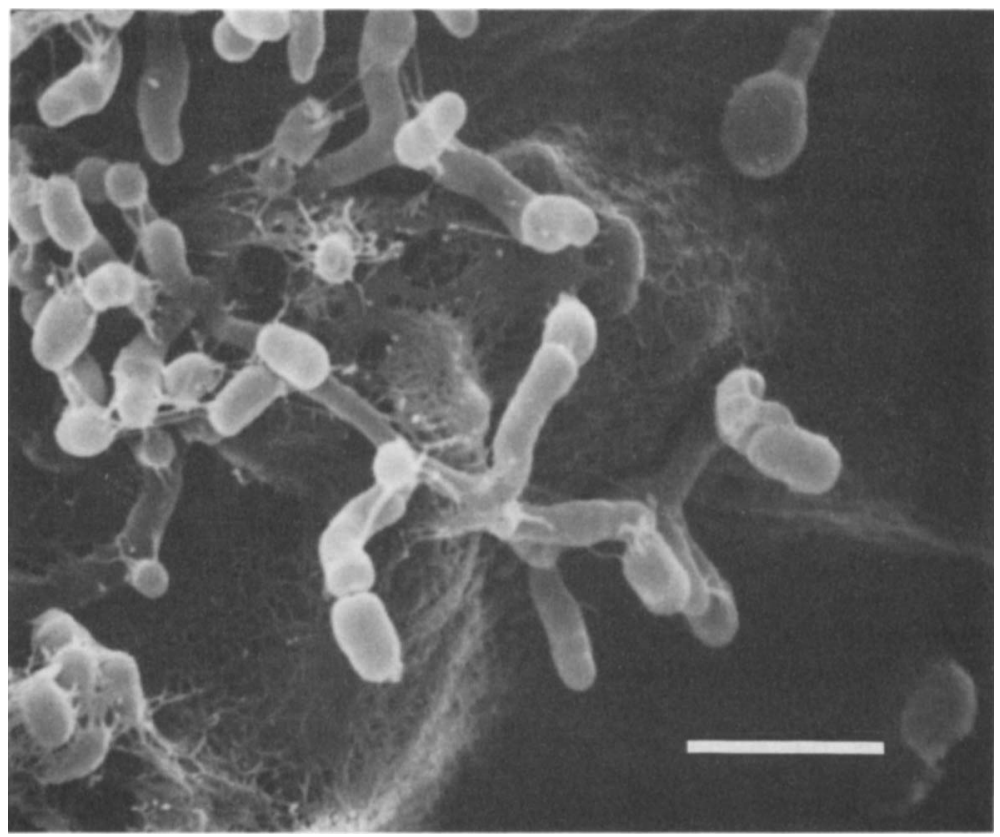

FIG. 1. Scanning electron micrograph of a 7-day culture on oatmeal agar. Single spores were formed at the tips of the substrate mycelia. $\mathrm{Bar}=2 \mu \mathrm{m}$.

defined, as pointed out by Williams et al. (22), and even actinomycetes with spore chains (e. g., Streptomyces spp.) bear spore sheaths. Furthermore, sporangium-forming actinomycetes are heterogeneous with respect to chemical data (3); the genera Microellobosporia, Elytrosporangium, and Kitasatoa have wall chemotype I, the genera Actinoplanes and Dactylosporangium and their relatives have chemotype IID, and the genera Streptosporangium, Planobispora, and Planomonospora have chemotype IIIB. Therefore, the organisms should be compared for their chemotaxonomic properties, as well with the properties of the other actinomycete genera.

$K$. aurantiaca had both $\mathrm{LL}$ - and meso- $\mathrm{A}_{2} \mathrm{pm}$ in its cell walls, lacked a characteristic sugar pattern, had phosphatidylcholine as a diagnostic phospholipid (type PIII), had MK-9( $\left(\mathrm{H}_{4}\right)$ as a main isoprenolog, and had a fatty acid profile lacking any iso/anteiso branched fatty acids. The two $\mathrm{A}_{2} \mathrm{pm}$ isomers are found in the walls of several Actinomadura (10), Micromonospora (6), and Kitasatosporia species strains (15, 16). The distribution of the $\mathrm{A}_{2} \mathrm{pm}$ isomers in Kitasatosporia

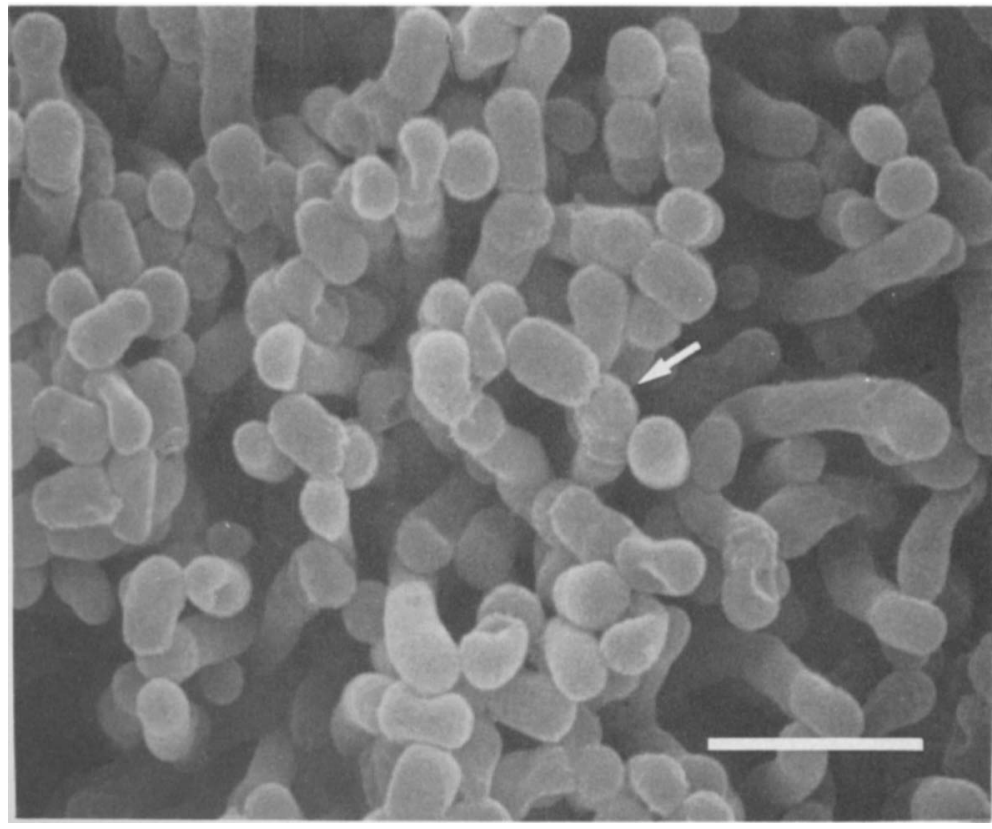

FIG. 2. Scanning electron micrograph of a 1-month culture on water agar: the surface of a central projection from above. Catenated spores are indicated by the arrow. The specimen was conductive stained and lightly coated with gold. Bar $=2 \mu \mathrm{m}$. 


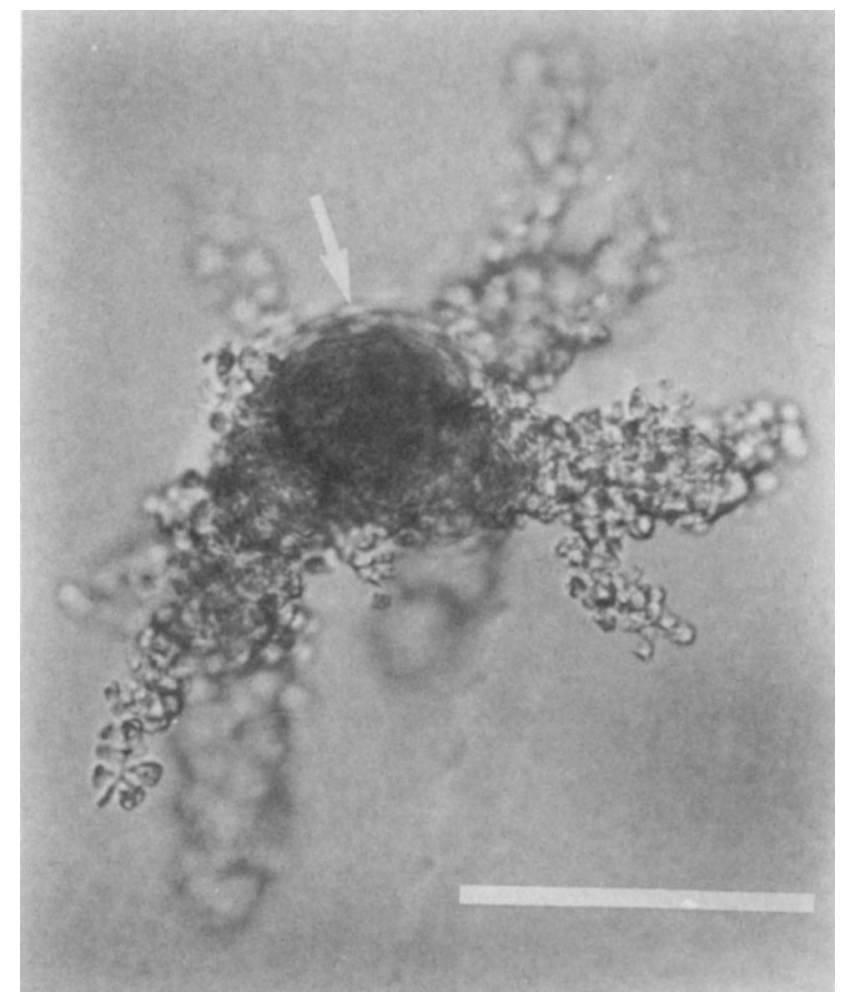

FIG. 3. Light micrograph of a colony which formed bunches of spore clusters on water agar ( 7 days). All structures, except a small dome projection (arrow), developed in the agar. Bar $=50 \mu \mathrm{m}$.

species strains, which contain meso- $\mathrm{A}_{2} \mathrm{pm}$ in their mycelia and LL-A $A_{2}$ pm in their spores $(15,16)$, is the reverse of the distribution of $\mathrm{A}_{2} \mathrm{pm}$ in $K$. aurantiaca. The strains of Actinomadura and Micromonospora species have only a trace of
LL- $\mathrm{A}_{2} \mathrm{pm}$, and it is not certain whether the occurrence of $\mathrm{LL}-\mathrm{A}_{2} \mathrm{pm}$ is due to the presence of submerged spores in the preparation. Among the actinomycetes investigated so far (3), phosphatidylcholine and $\mathrm{MK}-9\left(\mathrm{H}_{4}\right)$ (as a main menaquinone component) have been found in some mycolateless wall chemotype IV actinomycetes (e. g., Faenia and Saccharopolyspora spp.), whereas a fatty acid profile lacking iso/anteiso branched fatty acids is characteristic of mycolic acid-containing actinomycetes with cell wall type IV. Thus, the combination of the phospholipid, menaquinone, and fatty acid patterns of the test strain does not correspond to the pattern of any of known actinomycete genus. Grouping of aerobic actinomycetes based on cell wall amino acid composition and other chemotaxonomic properties reflects the phylogenetic relatedness deduced from $16 \mathrm{~S}$ ribosomal ribonucleic acid data $(3,13)$. The chemotaxonomic properties of $K$. aurantiaca might support a new line in the suprageneric classification of actinomycetes; however, the precise assignment of the genus Kineosporia at the suprageneric level should await further appropriate approaches.

In this study we revised the chemotaxonomic and morphological properties of $K$. aurantiaca, and our results support the contention that strain $\mathrm{JCM} 3230^{\mathrm{T}}$ constitutes a valid genus. However, it should be noted that the morphology of $K$. aurantiaca is quite similar to the morphologies of spore dome and spore head colonies described by Willoughby (23); the central (dome) projection and the spores occurring on an inflated phialidelike structure are termed spore dome and spore head, respectively. Makkar and Cross (9) also isolated the spore dome actinomycetes, which contained the two $\mathrm{A}_{2} \mathrm{pm}$ isomers, lacked a characteristic sugar pattern, and had MK-9 $\left(\mathrm{H}_{4}\right)$ as a main isoprenolog $(1,2,9)$. Thus, the situation of the spore dome actinomycetes matches that of $K$. aurantiaca in chemotaxonomic and the morphological aspects. It is interesting that the spore dome actinomycetes of Willoughby and of Makkar and Cross were isolated from freshwater habitats, whereas $K$. aurantiaca

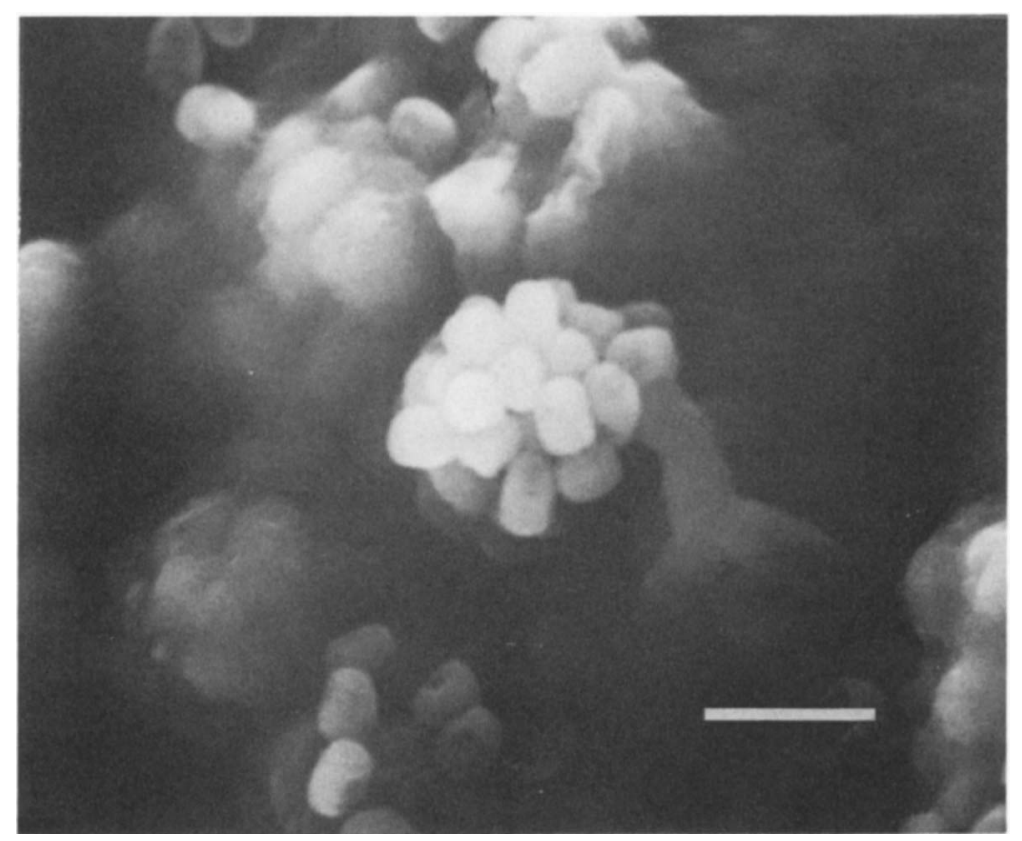

FIG. 4. Scanning electron micrograph of a 1-month culture on oatmeal agar. A spore cluster with a sporophore is shown. The specimen was conductive stained. Bar $=2 \mu \mathrm{m}$. 


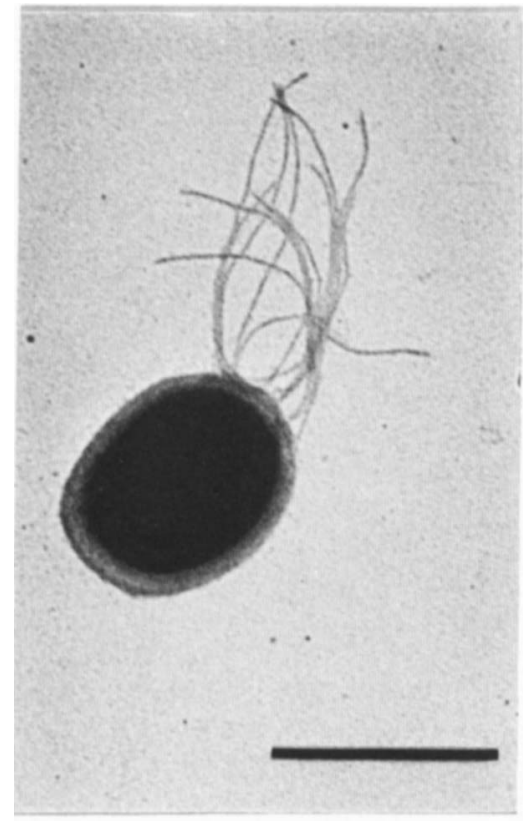

FIG. 5. Transmission electron micrograph of a zoospore with a polar tuft of flagella. Bar $=1 \mu \mathrm{m}$.

was isolated from soil. Cross and Alderson have used the provisional name "Cupolomyces" for the spore dome actinomycetes (1); however, these organisms could be accommodated within the genus Kineosporia. Preliminary numerical studies by Cross and Makkar (2) showed the heterogeneity within the spore dome actinomycetes, but further studies are needed to clarify the taxonomic status of $K$. aurantiaca and the spore dome actinomycetes.

At present, $K$. aurantiaca is the sole member of the genus, and the description of the genus Kineosporia is emended below.

Amended description of the genus Kineosporia Pagani and Parenti 1978. Aerobic, gram positive, and non-acid fast. Colonies on agar medium lack aerial mycelia, form central projections with radiating vegetative hyphae, and are occasionally accompanied by bunches of spore clusters in the agar. Mature colonies have a gelatinous matrix which confers a glossy appearance. Spores, which are spherical to ovoid or pyriform with a long axis of 1 to $2 \mu \mathrm{m}$, are catenated around the central projection or are located singly or aggregately at the tips of hyphae. The spores are motile with polar tufts of flagella.

The peptidoglycan contains both LL- and meso- $\mathrm{A}_{2} \mathrm{pm}$; the former, accompanied by glycine, is in the mycelium, and the latter is in the spores. The whole cultured organism contains galactose, glucose, mannose, and ribose, but the content of mannose is negligible in the spore fraction. Phosphatidylcholine is a diagnostic phospholipid, and $\mathrm{MK}-\mathbf{9}\left(\mathrm{H}_{4}\right)$ is present as a main menaquinone component. The cellular fatty acid

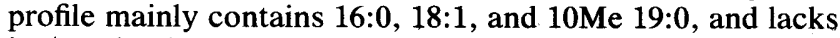
iso/anteiso branched fatty acids. 2-Hydroxy fatty acids are also present. Lipid compositions are not significantly different in the whole cultured organism and the spore fraction. Mycolic acids are absent. The guanine-plus-cytosine content of the deoxyribonucleic acid is $69.2 \mathrm{~mol} \%$.

$K$. aurantiaca is the type species, and the type strain is strain JCM 3230 (= ATCC 29727).

\section{ACKNOWLEDGMENT}

We thank M. Chijimatsu, RIKEN, Saitama, Japan, for analyzing the amino acid composition of the peptidoglycans.

\section{LITERATURE CITED}

1. Cross, T., and G. Alderson. 1988. What weight morphology in current actinomycete taxonomy?, p. 216-220. In Y. Okami, T. Beppu, and H. Ogawara (ed.), Biology of actinomycetes '88. Japan Scientific Societies Press, Tokyo.

2. Cross, T., and N. S. Makkar. 1986. Spore dome actinomycetes, p. 579-581. In G. Szabó, S. Biró, and M. Goodfellow (ed.), Biological, biochemical and biomedical aspects of actinomycetes. Akadémiai Kiadó, Budapest.

3. Goodfellow, M., and T. Cross. 1984. Classification, p. 7-164. In M. Goodfellow, M. Mordarski, and S. T. Williams (ed.), The biology of actinomycetes. Academic Press, Inc. (London), Ltd., London.

4. Hasegawa, T., M. Takizawa, and S. Tanida. 1983. A rapid analysis for chemical grouping of aerobic actinomycetes. J. Gen. Appl. Microbiol. 29:319-322.

5. Ikemoto, S., H. Kuraishi, K. Komagata, R. Azuma, T. Suto, and H. Murooka. 1978. Cellular fatty acid composition in Pseudomonas species. J. Gen. Appl. Microbiol. 24:199-213.

6. Kawamoto, I., T. Oka, and T. Nara. 1981. Cell wall composition of Micromonospora olivoasterospora, Micromonospora sagamiensis, and related organisms. J. Bacteriol. 146:527534.

7. Lechevalier, M. P., C. De Bievre, and H. Lechevalier. 1977. Chemotaxonomy of aerobic actinomycetes: phospholipid composition. Biochem. Syst. Ecol. 5:249-260.

8. Lechevalier, M. P., and H. Lechevalier. 1970. Chemical composition as a criterion in the classification of aerobic actinomycetes. Int. J. Syst. Bacteriol. 20:435-443.

9. Makkar, N. S., and T. Cross. 1982. Actinoplanetes in soil and on plant litter from freshwater habitats. J. Appl. Bacteriol. 52: 209-218.

10. Mordarska, H., M. Mordarski, and M. Goodfellow. 1972. Chemotaxonomic characters and classification of some nocardioform bacteria. J. Gen. Microbiol. 71:77-86.

11. Pagani, H., and F. Parenti. 1978. Kineosporia, a new genus of the order Actinomycetales. Int. J. Syst. Bacteriol. 28:401406.

12. Saito, H., and K. Miura. 1963. Preparation of transforming deoxyribonucleic acid by phenol treatment. Biochim. Biophys. Acta 72:619-629.

13. Stackebrandt, E. 1986. The significance of "wall types" in phylogenetically based taxonomic studies on actinomycetes, $p$. 497-506. In G. Szabó, S. Biró, and M. Goodfellow (ed.), Biological, biochemical and biomedical aspects of actinomycetes. Akadémiai Kiadó, Budapest.

14. Staneck, J. L., and G. D. Roberts. 1974. Simplified approach to identification of aerobic actinomycetes by thin-layer chromatography. Appl. Microbiol. 28:226-231.

15. Takahashi, Y., Y. Iwai, and S. Omura. 1983. Relationship between cell morphology and the types of diaminopimelic acid in Kitasatosporia setalba. J. Gen. Appl. Microbiol. 29:459465 .

16. Takahashi, Y., Y. Iwai, and S. Omura. 1984. Two new species of the genus Kitasatosporia, Kitasatosporia phosalacinea sp. nov. and Kitasatosporia griseola sp. nov. J. Gen. Appl. Microbiol. 30:377-387.

17. Tamaoka, J., Y. Katayama-Fujimura, and H. Kuraishi. 1983. Analysis of bacterial menaquinone mixtures by high performance liquid chromatography. J. Appl. Bacteriol. 54:31-36.

18. Tamaoka, J., and K. Komagata. 1984. Determination of DNA base composition by reversed-phase high-performance liquid chromatography. FEMS Microbiol. Lett. 25:125-128.

19. Toriyama, S., I. Yano, M. Masui, E. Kusunose, and N. Akimori. 1980. Regulation cell wall mycolic acid biosynthesis in acid-fast bacteria. J. Biochem. (Tokyo) 88:211-221.

20. Waksman, S. A. 1961. The actinomycetes, vol. 2, p. 328-334. 
The Williams \& Wilkins Co., Baltimore.

21. Watanabe, T., T. Nagatani, and T. Murakami. 1975. New non-coating observation techniques in SEM. Saibou 7:118-125.

22. Williams, S. T., G. P. Sharples, and R. M. Bradshaw. 1973. The fine structure of the Actinomycetales, p. 113-136. In G. Sykes and F. A. Skinner (ed.), Actinomycetales: characteristics and practical importance. Academic Press, Inc. (London), Ltd., London.

23. Willoughby, L. G. 1969. A study on aquatic actinomycetes, the allochthonous leaf component. Nova Hedwigia 18:45-113. 